\title{
Migraciones e informalidad urbana. Dinámicas contemporáneas de la exclusión y la inclusión en Buenos Aires ${ }^{1}$
}

Luciana Vaccotti. Universidad de Buenos Aires (UBA), Buenos Aires, Argentina.

RESUMEN | Este artículo aborda algunas articulaciones entre procesos migratorios y dinámicas de la informalidad urbana en la Ciudad Autónoma de Buenos Aires durante el periodo 2001-2014, en el marco de conflictos sociales que han vuelto a posicionar estos temas en la agenda política y mediática argentina. Se basa en los hallazgos de un estudio de caso, Playón de Chacarita, una villa o nuevo asentamiento urbano poblado mayoritariamente por migrantes, principalmente peruanos. La reconstrucción de su historia y dinámicas se nutre de los relatos de sus habitantes y otros actores clave, más bibliografía especializada. El análisis de esta configuración socioespacial concreta ofrece elementos para comprender la forma en que se conjugan y reactualizan dos procesos de exclusión históricamente entrelazados: uno vinculado a la clase social y otro al origen étnico-nacional. También habilita una reflexión sobre las estrategias que despliegan quienes sufren estas desigualdades para desdibujar estas fronteras y construir formas alternativas de inclusión.

PALABRAS CLAVE | migración, vivienda, mercado inmobiliario.

ABSTRACT | This paper pursues to analyze present articulations between migration and urban informality in the City of Buenos Aires (2001-2014), amid rising social conflicts that have brought these issues back to the political and mass media agendas in Argentina. This research presents the findings of a case study conducted at Playon de Chacarita, a slum located in Buenos Aires and mostly inhabited by immigrants, especially from Peru. The history and evolving dynamics of this space, as well as its relationship with the processes examined in this paper are reconstructed from the stories of its residents and other social actors, in dialogue with specialized literature. Studying this social and spatial configuration contributes to understanding how two historically linked exclusion processes - one based on social class and another based on ethnical and national origin-combine and actualize in the present. The paper also seeks to foster reflections on the strategies developed by the groups that suffer these inequalities to blur these borders: to build alternative forms of inclusion.

KEYWORDS | migration, housing, housing market.

Recibido el 27 de agosto de 2015, aprobado el 22 de junio de 2016

E-mail: luciana.vaccotti@gmail.com

1 Este estudio de caso fue desarrollado en el marco de la tesis del Doctorado en Ciencias Sociales de la Universidad de Buenos Aires, entre los años 2011 y 2014. La estrategia metodológica se basó fundamentalmente en métodos cualitativos: 31 entrevistas en profundidad (a delegados y referentes barriales, militantes de agrupaciones y referentes políticos), observación participante y no participante (en diversas instancias en el barrio y otros espacios), y análisis documental. 


\section{Introducción}

En América Latina, los estudios urbanos históricamente han privilegiado el análisis de los espacios de pobreza, dando cuenta de los rasgos compartidos que asumen en la región, así como de sus singularidades en cada sociedad y cada contexto sociopolítico específico. En Argentina existe una vasta producción académica sobre diversas cuestiones vinculadas con villas, asentamientos y otras expresiones locales de la informalidad urbana, en diálogo con las principales vertientes latinoamericanas.

Los estudios migratorios, por su parte, exhiben un desarrollo más dispar en la región, vinculado a las distintas realidades migratorias de los países y los cambiantes contextos regionales e internacionales que inciden en las formas de movilidad humana. En Argentina - una sociedad tempranamente marcada por intensas migraciones internacionales-, este campo de estudio muestra una amplia trayectoria y una gran vitalidad, que se expresa en una diversidad de centros y agendas de investigación. En las últimas décadas, las migraciones desde países limítrofes y Perú han ocupado un lugar destacado en los estudios migratorios, no solo debido a su relevancia demográfica, sino a las problemáticas derivadas de las posiciones que ocupan esas poblaciones en las jerarquías étnicas y sociales en el país.

La preocupación por la distribución espacial de los migrantes en las ciudades ha recorrido un largo camino desde los abordajes pioneros de la Escuela de Chicago a inicios del siglo xx. La bibliografía internacional aborda los factores que inciden en su asentamiento y localización diferencial fundamentalmente desde dos perspectivas: aquellas centradas en los migrantes y sus estrategias de asentamiento, y las que indagan en las condiciones socioeconómicas de la sociedad receptora. Estas últimas priorizan la cuestión de la vivienda -determinante de las pautas residenciales y las condiciones de vida de los migrantes- y los procesos discriminatorios en el acceso a ella (Mera, 2012).

Si bien este tema ha tenido escasa presencia en la historiografía y en los estudios migratorios y urbanos argentinos en general, en el periodo reciente se observa un creciente interés académico, motivado por la emergencia de conflictos sociales que han colocado en la agenda las interacciones entre procesos migratorios y dinámicas de la informalidad urbana. El presente artículo se inscribe en esta tendencia, a partir de las siguientes preguntas: ¿qué procesos inciden en la significativa concentración de población migrante en villas, nuevos asentamientos urbanos (NAU) y otras modalidades de informalidad urbana en Buenos Aires?; y ¿̨cuáles son los efectos de la presencia migrante en las dinámicas (sociales y urbanas) que se configuran en estos espacios?

Desde el punto de vista urbano, el Playón de Chacarita puede ser considerado una villa o un NAU. Las villas "de emergencia" o "miseria" constituyen la tipología más emblemática de la informalidad urbana argentina, por lo que han sido profusamente estudiadas. ${ }^{2}$ Se trata de urbanizaciones informales surgidas en la década de 1940, producto de ocupaciones de tierra urbana vacante, que -entre otras características- producen tramas urbanas irregulares; cuentan con una buena localización, 
en relación con los centros de producción y consumo; en Buenos Aires se asientan mayoritariamente en tierras fiscales; responden a una suma de prácticas individuales y diferidas en el tiempo; las viviendas son originalmente construidas con materiales precarios o de desecho; poseen una alta densidad poblacional; actualmente los pobladores son trabajadores poco calificados o informales; la composición de la población muestra la heterogeneidad de la pobreza, incluyendo a "antiguos" villeros, nuevos migrantes (del interior y de países limítrofes) y sectores pauperizados; y sus habitantes son portadores de adscripciones estigmatizantes (Cravino, 2006).

Los NAU, por su parte, son núcleos poblacionales surgidos en la década de 1990, ubicados en zonas intersticiales y no aptas para la urbanización, con elevados grados de precariedad, sin servicios, y no comprendidos dentro de ninguno de los planes previstos por el Estado para la radicación o urbanización. Estos espacios se encuentran en una situación de vacío administrativo: no existen políticas específicas para ellos (Lekerman, 2005). Al igual que otros espacios de estas características, el Playón de Chacarita surgió en la década de 1990. Se ubica en terrenos ferroviarios, contiguos a la estación Federico Lacroze del Ferrocarril Urquiza, emplazado entre las vías del ferrocarril y cuatro calles de un barrio porteño de clase media, Chacarita, donde ocupa una superficie algo inferior a las dos hectáreas. ${ }^{3}$ Los NAU han sido sensiblemente menos estudiados por las ciencias sociales. En este trabajo se opta por utilizar ambos términos (villa y NAU) como intercambiables, a los efectos de enfatizar la continuidad tipológica y social entre ambas figuras del hábitat popular argentino.

Desde la perspectiva migratoria, el Playón de Chacarita es un barrio que exhibe una población de origen marcadamente migrante, especialmente de procedencia peruana. La historia de la migración peruana hacia Argentina es más reciente que la de otros grupos de países vecinos. Este flujo adquirió relevancia en la década de 1990, a partir de la cual ha mostrado un fuerte dinamismo. Actualmente se trata del tercer colectivo más importante en la ciudad de Buenos Aires, y uno de los menos indagados.

En suma, este artículo se propone aportar elementos cualitativos a los debates actuales en torno a una serie de vacíos en los estudios urbanos y migratorios locales: (i) las formas de asentamiento de los migrantes en las ciudades argentinas, especialmente su inserción residencial en villas y NAU; (ii) las estrategias habitacionales específicas de los migrantes peruanos; (iii) las causas y consecuencias de la impronta migratoria de las villas y NAU; y (iv) el desarrollo y las características actuales de los NAU, y de uno en particular: el Playón de Chacarita.

\section{Migrantes en hábitats informales: rearticulaciones en la poscrisis}

En 2001, Argentina vivió un fuerte estallido social, seguido de una crisis económica, social y política sin precedentes. ${ }^{4}$ En la Ciudad Autónoma de Buenos Aires (CABA), las consecuencias de la crisis afectaron a amplios sectores, especialmente a

3 Varios estudios (Szajnberg, 2009; Rodríguez, Arqueros, Rodríguez, Gómez \& Zapata, 2011) consideran el Playón de Chacarita como un NAU.

4 El pBi se redujo en más del 11\%, el desempleo aumentó al 21,5\% y el porcentaje de la población viviendo con ingresos por debajo de la línea de pobreza alcanzó un techo histórico de 55\% (Beccaria, Maurizio \& Esquivel, 2005). 
los más desprotegidos, incluyendo a los migrantes internos y de países vecinos. Sin embargo, estas circunstancias no produjeron un retorno masivo de migrantes a sus países, aunque sí revirtieron la tendencia al aumento de estos flujos. Los migrantes sudamericanos permanecieron en el mercado laboral argentino, exhibiendo tasas de desocupación similares a los otros grupos (más bajas en el caso de las mujeres), a costa de aceptar peores condiciones de empleo. A tal flexibilidad contribuyó que parte de los migrantes continuó en situación de indocumentación, ya que las restricciones para la regularización migratoria persistieron aún después de la crisis (Maguid \& Arruñada, 2005).

Los migrantes peruanos aumentaron en un $430 \%$ en el periodo intercensal 1991-2001 (Rosas, Corbetta y Toledo, 2013). Hacia 2001 se localizaban principalmente en la САВA, donde su relevancia se ubicó cercana a la de los bolivianos y paraguayos (los colectivos migrantes más importantes en Argentina). En 2010, los peruanos representaban un 2,1\% de la población de la ciudad. La crisis de 2001 y sus consecuencias tampoco provocaron en ellos el retorno masivo a su país de origen, aunque sí afectaron significativamente sus condiciones de vida, incluyendo la dimensión sociohabitacional.

Estudios recientes que analizan los patrones de distribución espacial de los principales colectivos de migrantes en Buenos Aires, sugieren que los peruanos se encuentran más dispersos que los bolivianos y los paraguayos. ${ }^{5}$ Si bien se registra una tendencia al agrupamiento de las áreas de alta presencia de peruanos, estas se localizan más próximas al centro urbano, con las de mayor presencia relativa ubicadas principalmente en el este de la ciudad, en torno al Centro Administrativo y de Negocios. Esto da cuenta de una dinámica diferente de acceso al suelo por parte de este colectivo (Mera \& Marcos, 2015), una dinámica que ha sido observada también en otras ciudades latinoamericanas receptoras de migración peruana (Garcés, 2010).

En el Playón de Chacarita, el dinamismo sociodemográfico y la ausencia de estadísticas actualizadas, factores sumados a los problemas de subregistro que suelen afectar el conteo tanto de población migrante como de población residente en espacios informales, imposibilitan la construcción de un panorama preciso de su población. Según el Censo 2010, el barrio constaba de 585 viviendas, 727 hogares y 2.324 habitantes: 1.184 mujeres y 1.140 varones. Se trata de un barrio que exhibe una marcada impronta migratoria. Esta noción busca incorporar elementos surgidos de los estudios sobre lugares migrantes en la ciudad (Sassone, 2007), barrios étnicos y barrios de inmigrantes (Matossian, 2010; Sassone \& Mera, 2007), abordajes que no solo consideran la concentración residencial de los migrantes, sino también las sociabilidades que se desarrollan en las áreas en que residen, las formas de uso y

5 Los paraguayos, y especialmente los bolivianos, se encuentran muy desigualmente distribuidos y concentrados en el espacio urbano. Ambos grupos tienen una representación próxima a la media en la mayor parte de la ciudad, pero se concentran fuertemente en determinadas áreas (en algunas llegan a constituir más del $60 \%$ de la población). Asimismo, existe una tendencia a la agrupación de las áreas de alta presencia de estos grupos, las cuales no solo se encuentran alejadas del centro urbano sino que, en su mayoría, están ubicadas en el sur de la ciudad -la zona más degradada, con un mayor déficit de infraestructuras y servicios, y alta presencia de formas precarias de hábitat-o en áreas que contienen villas y asentamientos (Mera \& Marcos, 2015). 
apropiación del espacio urbano que expresan, y las prácticas culturales e identitarias que habilitan. Pensar en la impronta migratoria del Playón de Chacarita y otros barrios pobres de Buenos Aires implica considerar la fuerte presencia de migrantes y de prácticas vinculadas a ellos, que singularizan y visibilizan esos espacios no solo en su entorno barrial inmediato, sino en la ciudad en su conjunto.

El Playón de Chacarita es analizado en tanto expresión socioespacial concreta de las articulaciones entre migraciones e informalidad urbana configuradas en el periodo posterior a la crisis de 2001 y que, a su vez, se remontan a los procesos de incidencia mutua entre dichos fenómenos, consolidados a lo largo del siglo xx. Con la mediación del Estado, estos procesos se fueron plasmando en visiones y discursos hegemónicos, que fueron condensando a las "cuestiones" migratoria y villera en un único "problema” de renovada vigencia en el periodo que analiza este artículo: las villas pobladas por migrantes, o los migrantes que pueblan las villas. En otras palabras, el Playón de Chacarita no es solo una villa, ni solo un barrio de migrantes: es una villa con impronta migratoria; un "problema" que reviste mayor complejidad que un barrio precario o un barrio étnico, una "cuestión" que manifiesta las formas actualizadas en que se conjugan y refuerzan dos procesos de exclusión históricamente ligados en la ciudad de Buenos Aires: uno vinculado a la clase social y otro al origen étniconacional. Por un lado, el análisis de esta configuración socioespacial arroja elementos cualitativos para comprender de qué modo las desigualdades se articulan y sobredeterminan mutuamente, actualizando la relación entre condición migratoria y hábitat informal. Por otro lado, permite reflexionar acerca de las formas en las que estas nuevas expresiones socioespaciales son invariablemente construidas como "problemas".

Los últimos dos censos nacionales muestran que la población de las villas porteñas experimentó un aumento sostenido, alcanzando las 107.805 personas en 2001 y las 163.587 en 2010. La población extranjera, por su parte, creció en una tasa superior a la del total poblacional: en la última década aumentó un $18 \%$ en la población total del país y un $20 \%$ en la población de la ciudad. Según el Censo 2010, los colectivos de migrantes más importantes en la CABA son los paraguayos, los bolivianos y los peruanos, que en total representan un 7,6\% de la población de la capital argentina.

Por otro lado, la Encuesta Anual de Hogares (ЕAH) de 2012 señala que un 6\% de la población de la CABA reside en viviendas ubicadas en villas; un $4 \%$ en viviendas ubicadas en inquilinatos, hoteles familiares, pensiones e inmuebles usurpados/ tomados; y un $90 \%$ en viviendas ubicadas en el resto de la ciudad. O sea, un $10 \%$ de la población total de la ciudad vive en situaciones habitacionales precarias.

Si desagregamos la población de las villas de la ciudad según lugar de nacimiento, resulta que un $49 \%$ nació en la ciudad, un 34\% en un país limítrofe o en Perú, un $13 \%$ en otra provincia y un $3 \%$ en un partido del Gran Buenos Aires. ${ }^{6}$ Es decir, un $51 \%$ de la población de las villas porteńas es migrante. Ese porcentaje asciende a $64 \%$ en la población que vive en inquilinatos, hoteles, pensiones e inmuebles tomados, y a un $38 \%$ en el resto de la ciudad. La acentuada impronta migratoria de los contextos de informalidad urbana de la ciudad sin duda demanda 
que los análisis sobre estos espacios presten atención a sus imbricaciones con los procesos migratorios.

La ЕАH 2012 también revela que 56\% de los hogares en villas tienen un jefe o jefa de hogar que ha nacido en un país limítrofe o en Perú. En estos hogares, el porcentaje de "hijos, hijas, hijastros e hijastras" nacidos en la CABA asciende a un $69 \%$, frente a solo un $26 \%$ de aquellos nacidos en países limítrofes y en Perú. Este dato contribuye a la comprensión de la tendencia a magnificar la presencia de extranjeros en la ciudad y especialmente en los territorios de relegación. Si a las personas nacidas en países limítrofes y en Perú que habitan en las villas (el 34\% de la población de estos espacios), le sumamos a sus hijos ( $y$, en menor volumen, a sus nietos) nacidos en la CABA (y contabilizados correctamente como argentinos), obtenemos un volumen mayor de personas que son percibidas como extranjeras; que son visibilizadas y homogeneizadas étnicamente (Grimson, 2011), en el contexto de la impronta migratoria de estos espacios.

En este punto resulta inevitable hacer mención a los sucesos de 2010 en el Parque Indoamericano: los hechos de violencia que se produjeron durante esa toma de terrenos ${ }^{7}$ pusieron de manifiesto la persistencia de problemáticas sociales en las que se combinan procesos migratorios y dinámicas de informalidad urbana en Buenos Aires. Estos episodios tuvieron lugar en un contexto en el que amplios sectores de la población se vieron desplazados a las villas, debido a su incapacidad de acceder a una vivienda en el mercado formal, a la ausencia de programas de vivienda, al desfinanciamiento de los existentes, y a la nula inversión estatal en el mejoramiento de estos barrios. Sin embargo, funcionarios públicos y referentes políticos manifestaron lecturas discriminatorias de estos sucesos, visiones que responden a construcciones simbólicas de larga data y profundamente enraizadas en los discursos dominantes. Es fundamental establecer que no existe un vínculo relevante entre el déficit habitacional argentino y la migración limítrofe: ese déficit es muy superior a la cantidad de migrantes y, además, no todos los migrantes lo integran automáticamente (Grimson \& Caggiano, 2012).

El conflicto en el Parque Indoamericano y los que se sucedieron durante los años siguientes recibieron la atención inmediata del sector académico, que no dudó en tratar esta particular intersección entre procesos migratorios y dinámicas de la informalidad urbana como un problema sociológico, rompiendo así con el vacío que se observaba hasta ese momento en la producción académica respecto de tal realidad. En el periodo reciente diversos estudios realizados desde varias disciplinas de las ciencias sociales sugieren la emergencia de una incipiente línea de investigación. ${ }^{8}$

7 Un grupo de argentinos y migrantes, particularmente de Bolivia y Paraguay, ocuparon ese predio público, y se convirtieron en víctimas de una brutal represión por parte de fuerzas policiales y presuntos representantes de otros vecinos del barrio, con un saldo de tres personas muertas.

8 Algunos ejemplos son Gallinati y Gavazzo (2011), Caggiano et al. (2012), Mera (2012), Canelo (2013), Mera y Vaccotti (2013), Rosas, Corbetta y Toledo (2013), Gago y García Pérez (2014), Vaccotti (2014), Mera y Marcos (2015), entre otros. 


\section{El Playón de Chacarita: génesis y devenir de un espacio de pobreza}

Entre los principales procesos que empujaron al surgimiento y desarrollo del Playón de Chacarita y otros barrios de características similares durante la década de 1990, se cuenta el empobrecimiento general de la población, consecuencia de la implementación de políticas neoliberales que llevaron a niveles dramáticos de desocupación y subocupación, que desencadenó la crisis de 2001.

Desde 1990, la ciudad también asistió a un proceso continuo de endurecimiento de la legislación sobre desalojos forzosos, de la mano de una ausencia de acciones políticas coordinadas para la reducción del déficit habitacional. Así, importantes sectores de la población, fuertemente abatidos por la crisis y con crecientes necesidades habitacionales, se encontraron ante un Estado local que solo ofrecía soluciones paliativas, que los forzaban a permanecer en el espectro de la informalidad urbana.

Los terrenos donde se localiza el Playón de Chacarita pertenecen al Estado nacional, pero fueron administrados por empresas en el marco de la privatización de la actividad ferroviaria en la década de 1990. Los primeros habitantes de este espacio fueron empleados ferroviarios y sus familias, en su mayoría migrantes internos. Ante la imposibilidad de cubrir un conjunto de gastos vinculados con la vivienda de sus empleados, la empresa ofreció vagones en desuso, emplazados en el playón ferroviario y provistos de servicios (instalados informalmente por la empresa) para ser utilizados como viviendas. Durante esa década, la población del barrio se mantuvo reducida - una veintena de familias-y relativamente homogénea en términos sociales: argentinos, migrantes internos y empleados ferroviarios. El incipiente asentamiento pasaba desapercibido, ya que se encontraba rodeado por muros.

El Playón de Chacarita comenzó a recibir nuevos habitantes desde poco antes del estallido de la crisis de 2001. Muchos de los nuevos pobladores eran migrantes internos, que abandonaban sus provincias de origen debido al creciente desempleo. También comenzó a instalarse un nuevo grupo de habitantes, que devendría emblemático de la Argentina de la crisis: los cartoneros. ${ }^{9}$ Estas personas, que provenían mayoritariamente del Gran Buenos Aires, venían a trabajar a la capital y pasaban la noche en casillas precarias en este y otros asentamientos que, en esos años, se multiplicaban en la ciudad.

A partir de 2003, la economía argentina comenzó a mostrar signos de mejora, con un aumento sostenido del producto a un ritmo elevado ( $8,5 \%$ en promedio) durante cinco ańos consecutivos (2003-2007). Los indicadores de empleo mostraron notables progresos: el desempleo pasó de 22\% en 2002 a 16,4\% en 2003 (Cerrutti \& Grimson, 2004). También disminuyeron la pobreza y la indigencia (Baer, 2008). Además, el gobierno de Kirchner ${ }^{10}$ implementó una amplia gama de políticas habi-

9 Si bien la actividad laboral conocida como cartoneo -que consiste en la recuperación informal de residuos- se expandió exponencialmente a partir de la crisis de 2001, aún se encuentra fuertemente estigmatizada, exhibe niveles de informalidad extremos y carece de reconocimiento social (Dimarco, 2007).

10 Néstor Kirchner asumió como presidente en 2003, dando inicio a un movimiento político denominado kirchnerismo y continuado por su esposa, Cristina Fernández, quien triunfó en las elecciones presidenciales de 2007 y 2011. 
tacionales, orientadas a la situación de "emergencia" social y a la reactivación económica mediante el sector de la construcción. Así, mientras que la década de 1990 se caracterizó por la ausencia de políticas habitacionales sólidas -en un proceso general de reforma del Estado que apuntó a la mercantilización-, la crisis de 2001 abrió las puertas a una cierta reformulación de la situación (Ferraudi Curto, 2011). No obstante, esa mejoría económica e institucional que comenzó a vivir el país a partir de 2003 no implicó una ausencia de contradicciones en los temas que analiza este artículo.

\section{Las experiencias de los migrantes}

\section{Nuevo espacio, nuevas oportunidades}

En el Playón de Chacarita, 2005 aparece como un punto de inflexión: ese año comenzaron a instalarse migrantes peruanos en el barrio, en una tendencia que no haría más que profundizarse durante los años siguientes. Los factores que explican la llegada de migrantes a este espacio emergente son varios.

Por un lado, las villas más consolidadas de la ciudad habían experimentado un fuerte crecimiento como resultado de los procesos expuestos más arriba, por lo que muchas personas que se vieron empujadas hacia opciones habitacionales informales optaron por barrios en formación, como el Playón de Chacarita.

Los primeros migrantes que se establecieron en el barrio accedieron a sus viviendas mediante la compra de terrenos. Estas operaciones fueron propiciadas por los cartoneros y los trabajadores ferroviarios que residían previamente en el barrio y que, en el contexto de cierta mejoría económica, regresaban a sus lugares de origen. Los cartoneros, puntualmente, recorrían la ciudad desempeñando sus actividades; en esos itinerarios, interactuaban con otros grupos que padecían los efectos de la crisis, como los migrantes, entre quienes difundían la existencia de terrenos "a la venta” en el Playón de Chacarita. Estas operaciones dieron inicio al funcionamiento de un mercado inmobiliario informal en el barrio.

En América Latina, los submercados inmobiliarios informales han sido analizados en algunas ciudades de Brasil (Abramo, 2003, Baltrusis 2004) y México (Jiménez Huerta, 2000). En diálogo con esta bibliografía, Cravino (2006) define el mercado inmobiliario informal que se desarrolla en las villas argentinas como aquellas transacciones inmobiliarias mercantilizadas que no se ajustan a las normas legales y urbanas, que no se adaptan estrictamente a la lógica económica formal de la comercialización de inmuebles, y cuyos protagonistas interactúan por fuera de las regulaciones económicas legales. La ilegalidad de estas transacciones no implica su ilegitimidad sino que, por el contrario, la operación se apoya en la legitimidad que implica la ocupación del suelo o la construcción de la vivienda, que puede ser vendida o alquilada. Desde el punto de vista económico, estas transacciones no pueden ser analizadas como una mera expresión de la lógica de la ganancia, sino que interactúan además con otras lógicas, expresando así también una "renta social". Los productos que se transan no se ajustan a las normas de uso del suelo urbano, lo que se suma a una importante tendencia a la transformación dinámica de los inmuebles y a una ausente o deficitaria infraestructura. En estas operaciones no 
participan agentes intermediarios típicos, sino que las redes sociales en los barrios o los contactos cara a cara entre los habitantes de las urbanizaciones informales tienen una mayor relevancia.

Si bien es importante comprender las lógicas específicas del funcionamiento del mercado inmobiliario informal de villas y NAU, es fundamental conceptualizar adecuadamente sus lazos con otras lógicas formales, no solo inmobiliarias, sino también laborales. No se trata de dos ciudades - una formal y una informal- sino de una sola, cuyas lógicas formales e informales se articulan en esquemas que, en última instancia, contribuyen a la reproducción de la lógica capitalista. El mercado inmobiliario formal impone fuertes barreras a la entrada de los migrantes: precios elevados, exigencia de garantías de personas vinculadas que cuenten con propiedades (localizadas únicamente en la CABA), documentos argentinos y certificados de trabajo formalizado (Gallinati \& Gavazzo, 2011): un conjunto de requisitos de difícil cumplimiento para los migrantes que viven en el Playón de Chacarita y otras villas y NAu de la ciudad.

El Estado local, a través de sus diferentes agencias, desempeñó un papel central en la conformación del Playón de Chacarita y otros nuevos espacios de pobreza en la ciudad. El contexto analizado se vio marcado por un aumento de los desalojos forzosos -especialmente en casas tomadas ${ }^{11}$ - en los que se negociaba la vulneración del derecho a la vivienda a cambio de subsidios. Los montos de estos subsidios no respondían a ningún criterio habitacional, sino a la capacidad de presión de los grupos afectados. Por ende, no garantizaban el acceso a una vivienda, sino que operaban como un factor pacificador de los desalojos o como un estímulo para la radicación fuera del ámbito de la CABA, que consumía recursos y reproducía la informalidad que alimentaba el círculo de la emergencia habitacional (Di Filippo, 2010).

No todos los migrantes que accedieron a una vivienda en el Playón de Chacarita en esa época lo hicieron mediante este tipo de transacciones; también se produjeron tomas de tierra dentro del predio. Varios estudios señalan la institucionalización de las ocupaciones de tierras como estrategia de acceso al suelo urbano de los sectores populares en Argentina (Cravino, 2008) y en otros países de la región (Abramo, 2003, Merklen, 1999, entre otros).

El aumento exponencial de la población y de las edificaciones del Playón de Chacarita comenzó en 2007, periodo a partir del cual se observa una multiplicación de ocupaciones en espacios intersticiales de la ciudad (Cravino, 2012). Esta temporalidad coincide con el inicio de la primera gestión macrista ${ }^{12}$ en el gobierno

11 Así se denomina localmente a viviendas públicas o privadas abandonadas, piezas de inquilinatos "intrusadas" por el cese de pago, depósitos o fábricas cerradas, u otros lugares ociosos de la ciudad, que son ocupadas por individuos o familias de sectores populares que ingresan a ellos de forma ilegal y organizan allí su vida cotidiana (Rodríguez, 2005).

12 Mauricio Macri, del partido político Propuesta Republicana (PRo), accedió a la Jefatura de Gobierno de la CABA en 2007 y fue reelecto para ese cargo en 2011. Fue elegido Presidente de la Argentina en las elecciones nacionales de 2015. Los impactos de la llegada de esta coalición política al gobierno nacional en los temas que analiza este artículo son incipientes pero significativos, por lo que ciertamente demandan análisis. 
local, y con el rumbo de la política habitacional que este gobierno fue instalando y consolidando en la ciudad.

Por último, es preciso destacar que los migrantes peruanos introdujeron un cambio notorio en el Playón de Chacarita: comenzaron a construir con materiales de calidad en un barrio donde, hasta entonces, predominaban las viviendas precarias. Esto responde a que, para amplios sectores -incluyendo a los migrantes- estos barrios nacientes representaban una oportunidad de resolver de forma duradera las necesidades habitacionales que afectaban a un arco cada vez mayor de familias. Esta oportunidad, no obstante, implicaba disputas con otros grupos. Algunas investigaciones locales han notado que, en los contextos de crisis, la escasez de recursos (como la tierra y la vivienda) y las disputas cotidianas por el acceso a los mismos, suelen articularse con una creciente xenofobia (Grimson, 2009).

\section{Una ciudad doblemente excluyente}

La noción de estrategias habitacionales desarrollada por los estudios urbanos designa las decisiones que toman las familias o unidades domésticas y los objetivos que persiguen en materia de hábitat, y se definen en la intersección entre las necesidades y expectativas habitacionales de los hogares, y los condicionantes estructurales. Forman parte de las trayectorias residenciales (Di Virgilio \& Gil y De Anso, 2012): un concepto que alude a la relación entre movilidad social, territorial y habitacional de los miembros de un hogar desde el momento en que su jefa o jefe toma la decisión de migrar de su lugar de nacimiento o, si es persona nativa de la capital o del Gran Buenos Aires, del barrio anterior (migraciones intraurbanas) (Di Virgilio, 2007).

En las ciudades argentinas, los sectores populares -incluyendo a los migrantescomparten trayectorias residenciales marcadas por las siguientes características: precariedad de las viviendas y de la infraestructura urbana, informalidad dominial y urbana, inseguridad física (proveniente de la precariedad y la informalidad), e inestabilidad de las diferentes etapas de estas trayectorias. Los factores que condicionan estas trayectorias son múltiples.

Para los migrantes, especialmente para los recién llegados a la capital argentina, el acceso a la vivienda no solo se da en el marco de modalidades habitacionales precarias e informales, sino que se articula con formas igualmente precarias e informales de inserción laboral. Numerosos estudios explican la consolidación de un patrón de inserción laboral segmentada, que circunscribe a los migrantes a determinados sectores de la economía, como la construcción, las pequeñas industrias y el servicio doméstico, rubros históricamente marcados por la informalidad (Benencia, 2012). Esta tendencia también ha sido registrada por investigaciones realizadas en otras ciudades latinoamericanas (Madero \& Mora, 2011). El vínculo entre estas dos modalidades informales de inserción social se manifiesta en el hecho ya citado de que la documentación probatoria de ingresos constituye un requisito común para el acceso al mercado inmobiliario formal.

Las trayectorias residenciales señaladas conllevan un conjunto de aprendizajes, de sentidos construidos que inciden en las estrategias habitacionales de los individuos y las familias. Los habitantes del Playón de Chacarita y de otros espacios de pobreza urbana mencionan dos certezas a las que arribaron en el curso de estas trayectorias: 
la necesidad de dejar de alquilar (considerada la única forma posible de ahorro y movilidad social en un contexto de bajos ingresos) y la importancia de contar con un lugar seguro y estable para vivir.

Entre las personas que viven en esta y otras villas de Buenos Aires y otras ciudades argentinas se observa un fuerte deseo de convertirse en "propietarios". Esta expectativa se expresa en las trayectorias y formas de movilidad social disponibles para estos sectores: recorridos con características comunes que, en el marco de los procesos migratorios, evidencian singularidades. Muchas de estas trayectorias comienzan con el alquiler de una habitación y finalizan con la compra (y posterior mejora) de una vivienda en el mercado inmobiliario informal.

Los migrantes no son ajenos a la expectativa de convertirse en propietarios. Si bien reconocen los aspectos (materiales y simbólicos) negativos que implica vivir en una villa, evalúan que se trata de la única estrategia posible para convertirse en propietarios de una vivienda (y, eventualmente, del terreno en el que esta se erige), en el escenario excluyente que se ha venido configurando en la ciudad.

La opción de vivir en una villa implica diversos riesgos. Por ejemplo, en el proceso de comprar un terreno y edificar una vivienda en una villa es frecuente que se produzcan usurpaciones. Esto es posible debido a la presencia diferencial de las fuerzas de seguridad en estos espacios, que deja librada la resolución de conflictos interpersonales a sus propios protagonistas. La falta de seguridad jurídica de la tenencia de las viviendas y las tierras expone a los habitantes de las villas a una fuerte desprotección. Este y otros ejemplos permiten dimensionar la versatilidad de las formas en que el Estado contribuye a la perpetuación de las condiciones de vida en dichos barrios.

Las trayectorias residenciales de los grupos aquí estudiados se desarrollan en un escenario local marcado por las políticas aplicadas por las gestiones del partido político (PRO). A partir de 2007, cuando se dio inicio a la primera administración de esta fuerza política, comienza a observarse una disminución en las asignaciones presupuestarias destinadas a la vivienda, que se acentuó en los años siguientes. También se produjo un proceso de "desguasamiento" de las políticas de hábitat, incluyendo a aquellas destinadas a las villas (Rodríguez et al., 2011). La gestión macrista ha debilitado los marcos institucionales y de legalidad impulsados por los sectores populares e instrumentales a sus intereses. Esto incluye el incumplimiento de varias leyes (el artículo 31 de la Constitución de la Ciudad, ${ }^{13}$ la Ley Nro. $148^{14}$

13 "La Ciudad reconoce el derecho a una vivienda digna y a un hábitat adecuado. Para ello: 1. Resuelve progresivamente el déficit habitacional, de infraestructura y servicios, dando prioridad a las personas de los sectores de pobreza crítica y con necesidades especiales de escasos recursos. 2. Auspicia la incorporación de los inmuebles ociosos, promueve los planes autogestionados, la integración urbanística y social de los pobladores marginados, la recuperación de las viviendas precarias y la regularización dominial y catastral, con criterios de radicación definitiva. 3. Regula los establecimientos que brindan alojamiento temporario, cuidando excluir los que encubran locaciones". Constitución de la Ciudad de Buenos Aires, art. 31 (1 de octubre de 1996). Boletín Oficial de la Ciudad Autónoma de Buenos Aires Nro. 47. http://www.buenosaires.gob.ar/areas/ leg_tecnica/sin/normapop09.php?id=26766\&qu=c\&ft=0\&c

14 Ley 148 , de atención prioritaria a la problemática social y habitacional en las Villas y los Núcleos habitacionales transitorios (1998). http://www2.cedom.gob.ar/es/legislacion/normas/leyes/ley148.html 
y la Ley Nro. 341, ${ }^{15}$ entre otras) promovidas en el marco de procesos participativos y sancionadas durante la segunda mitad de la década de 1990, en el contexto de la autonomización política de la CABA (1996). Como corolario de las dos gestiones macristas analizadas, ${ }^{16}$ la política pública hacia las villas quedó fragmentada entre varios organismos. Esto representa un obstáculo adicional para la implementación de las políticas de regularización, afectadas por una definición que enfatiza más la cuestión del suelo o la obra pública que la de las personas y las características históricas, sociales, organizativas y culturales de los procesos habitacionales.

Durante estas gestiones también se observan medidas que muestran que "algunas de las principales iniciativas orientadas a las urbanización de villas tienen mucha relación con el accionar del Poder Legislativo y el Judicial y poca (por no decir nada) con el Poder Ejecutivo" (Rodríguez et al., 2011, s/p). ${ }^{17}$

Los desalojos que se venían produciendo desde hacía años no hicieron más que pronunciarse con la llegada y la consolidación del macrismo en el gobierno, en el marco del afianzamiento de una "lógica de la expulsión" en la ciudad (Centro de Estudios Legales y Sociales [CELS], 2009). Sin embargo, el Estado local no es el único actor que participa de estos procesos; por el contrario, comparte protagonismo con agentes inmobiliarios, propietarios privados y con el propio Estado Nacional.

Entre las causas del arribo masivo de migrantes peruanos al barrio se cuenta el deterioro general de la situación en las casas tomadas, fundamentalmente el aumento de los desalojos forzosos. Numerosos migrantes de esta nacionalidad accedieron a esta modalidad habitacional durante la década de 1990 y comienzos de los 2000, con dos grandes consecuencias para ellos: fueron estigmatizados, al tiempo que experimentaron un intenso proceso de aprendizaje.

La situación de los espacios definidos como NAU continuó tornándose crítica con el paso de los años. Sin embargo, en el periodo analizado no se observaron canales de diálogo institucionalizados entre los distintos niveles de gobierno, ${ }^{18} \mathrm{ni}$ políticas públicas destinadas a atender la problemática específica de estos barrios. Por el contrario, se registró una perpetuación de la precariedad de las condiciones de vida de ellos e, incluso, un empeoramiento de las mismas, generado por la falta de coordinación entre el gobierno local y el gobierno nacional para atender la situación (Di Filippo, 2010).

Otro aspecto importante de señalar es que las políticas urbanas y habitacionales del pro han ido de la mano del desarrollo de ciertas capacidades del "clientelismo asistencial" y de la expansión de redes sociales de asistencia, "como contrapartida compensatoria a los más vulnerables” (Rodríguez et al., 2011, s/p). Esta cuestión aún no ha sido debidamente estudiada.

15 Ley 341 -CABA- de Cooperativas y Viviendas (24 de febrero de 2000). http://moi.org.ar/wpcontent/uploads/2015/10/Ley-341-CABA-Cooperativas-y-Viviendas.pdf

16 La tercera gestión de esta fuerza política en la ciudad, conducida por Horacio Rodríguez Larreta, muestra una clara línea de continuidad en materia de políticas destinadas a villas y NAU.

17 Este tema es abordado en profundidad en Vaccotti (2017).

18 En ese entonces, de distinto signo político; actualmente ambos niveles son gobernados por la alianza política Cambiemos. 
Por último, es preciso dejar asentado que los obstáculos que enfrentan los migrantes para acceder a la vivienda también son de orden simbólico. Las experiencias de los migrantes que residen en el Playón de Chacarita y en otras villas y NAU dan cuenta de los prejuicios de la clase propietaria en su contra, del modo en que la xenofobia también dificulta el acceso a una vivienda en el mercado formal, incluso cuando los migrantes logran cumplir con los requisitos establecidos por este.

\section{La incidencia de los recursos sociales}

Las redes y el capital social cumplen un papel fundamental en las estrategias de reproducción social de los sectores populares (Gutiérrez, 2008; Hintze, 2004). En el campo de los estudios migratorios, durante la última década han surgido varios trabajos abocados al análisis de las redes migratorias. Analizar los flujos migratorios desde este enfoque teórico-metodológico implica estudiar un proceso colectivo que involucra a varias generaciones del grupo doméstico, en el que las decisiones están condicionadas por elementos tanto materiales como simbólicos (Pedone, 2003). Estas redes no son ni espontáneas ni efímeras, sino que se transforman y complejizan con el tiempo debido a las relaciones que genera la entrada de otros actores dentro de su estructura. Estudiar cómo están compuestas estas redes y qué formas adoptan resulta clave para comprender el acceso a las oportunidades por parte de los migrantes (Benencia, 2005).

Las redes de familiares, conocidos y paisanos desempeñan una función fundamental en muchos aspectos clave del proyecto migratorio, especialmente en lo que respecta a trabajo y vivienda en el lugar de destino. Esto ha sido señalado por estudios nacionales e internacionales. Los familiares o paisanos prestan una ayuda crucial a los migrantes para el acceso a la vivienda al momento de la llegada al país, y para cambiar de vivienda a lo largo de sus trayectorias residenciales. En el mercado inmobiliario informal de las villas, las redes sociales -incluyendo las migratoriasocupan un lugar central: algunas investigaciones incluso registran una fuerte relación entre la nacionalidad de propietarios y de inquilinos en estos barrios (Cravino, 2011). A los alquileres se accede por la información que circula de "boca en boca" en el lugar mismo, por lo que las redes resultan centrales.

Los recursos sociales revisten una importancia clave para el conocimiento y el acceso de los migrantes peruanos al Playón de Chacarita y a otros barrios. Las articulaciones entre redes familiares, de paisanos y territoriales posibilitan la concreción de los proyectos habitacionales. Estos intercambios de capital social son dinámicos: se actualizan constantemente, en la medida en que también lo hacen las redes sociales. Los migrantes que alguna vez recibieron ayuda para acceder a la vivienda, posteriormente brindan asistencia a otros migrantes, movilizando su propio capital social en el marco de sus redes.

La cohabitación muestra una fuerte presencia en las estrategias habitacionales que forman parte de las experiencias migratorias, no solo en Buenos Aires sino en otras ciudades latinoamericanas (Segura \& Abde, 2014). Si bien esta estrategia responde a la crisis económica y a la necesidad de compartir gastos, es importante recordar que en el marco de las experiencias migratorias, la residencia con familiares y conocidos implica también otros significados (Di Virgilio \& Gil y de Anso, 2012). Al respecto, 
las redes sociales no solo contribuyen con cuestiones de subsistencia, como el acceso a la vivienda y al trabajo: la concentración territorial de los migrantes en ciertos barrios también actualiza las dinámicas de su sociabilidad. Las redes sociales de los migrantes establecen vínculos con el espacio, que se expresan en prácticas de identificación y comerciales. En este contexto, el Playón de Chacarita asiste a un conjunto de cambios en la sociabilidad barrial vinculados a la composición migratoria de la población. Las actividades y emprendimientos gastronómicos se sitúan entre los ejemplos más salientes de esta tendencia: en los últimos años se multiplicó la oferta de comida peruana dentro del barrio. También se realizan festejos religiosos y culturales tradicionales de Perú. Estas cuestiones han sido registradas por investigaciones realizadas en Buenos Aires (Grimson, 2011; Sassone \& Mera, 2007), otras ciudades argentinas (Matossian, 2010) y latinoamericanas (Arias, Moreno \& Núñez, 2010).

Otro aspecto determinante de la sociabilidad que se teje en el barrio es que sus habitantes se vinculan mayoritariamente con personas de su misma nacionalidad. Estimaciones propias, basadas en la Encuesta Anual de Hogares (EAH, 2012), arrojan que en el 83\% de los hogares en villas en los que el jefe o jefa de hogar nació en un país limítrofe o en Perú, los cónyuges o parejas también provienen de esos países (aunque no es posible establecer si son oriundos del mismo país o de otro de los que integran esta categoría). Un hallazgo significativo del trabajo de campo en el Playón de Chacarita es que muchas parejas de migrantes se formaron en Argentina. Este dato permite dimensionar las múltiples formas en que las redes migratorias y los procesos de exclusión a las que ellas responden moldean las experiencias de los migrantes. El escaso vínculo entre los migrantes peruanos y los argentinos ha sido señalado por otras investigaciones (Rosas, 2010).

\section{Un barrio, muchos sentidos}

Los migrantes enfrentan múltiples prejuicios para el acceso a los mercados formales de la vivienda y el trabajo en Buenos Aires. Pero los preconceptos en su contra no provienen únicamente de actores como los propietarios de inmuebles, los empleadores o el propio Estado a través de sus diferentes agencias: son reproducidos también por ellos mismos. Los migrantes que viven en el Playón de Chacarita mayoritariamente provienen de otras experiencias habitacionales precarias, como las casas tomadas. En el marco de las transformaciones atravesadas por la ciudad y la consolidación de su carácter crecientemente excluyente, numerosos migrantes peruanos debieron considerar por primera vez la posibilidad de vivir en una villa. Esa decisión implicó un proceso de resignificación parcial de estos espacios, uno de relativa superación de prenociones aprendidas en el transcurso de sus experiencias en la Argentina.

En paralelo a ese proceso de resignificación parcial, los habitantes del Playón de Chacarita despliegan un conjunto de estrategias que buscan disputar los sentidos negativos hegemónicos atribuidos al barrio y a ellos mismos (en tanto migrantes que habitan un espacio estigmatizado). Las principales acciones involucran la movilización política, y serán analizadas en otra ocasión. También se observan estrategias más cotidianas, como la permanente mejora de las viviendas, a través de la cual los habitantes del barrio canalizan sus expectativas de progreso social. En general, los 
discursos de los habitantes de este y otros barrios constantemente buscan distanciarse de algunos de los sentidos estigmatizantes atribuidos a las villas y a sus pobladores.

Estos dos procesos -la resignificación parcial del barrio y la disputa simbólica en la que se embarcan sus habitantes- son habilitados por la consolidación del barrio. Este afianzamiento responde a dos factores: por un lado, el aumento de la población y las edificaciones descarta la posibilidad de un eventual desalojo, uno de los principales temores de las personas que han atravesado experiencias de este tipo; por otro, la movilización política del barrio ha resultado en un cierto reconocimiento estatal, que también permite pensar en posibles soluciones a su situación de inseguridad jurídica.

Desde un punto de vista más instrumental, el barrio es elegido por su ubicación y por las externalidades que ofrece. ${ }^{19} \mathrm{El}$ fácil acceso a medios de transporte que lo comunican con los lugares de interés - principalmente vinculados al trabajo- se cuenta entre los aspectos más valorados a la hora de escoger este lugar. Los habitantes del Playón de Chacarita, especialmente los migrantes, ven el barrio como una puerta de entrada a la ciudad (Mera, 2012): un lugar en el que pueden instalarse para, desde allí, acceder a sus distintas actividades cotidianas y a los servicios que ofrece Buenos Aires. ${ }^{20}$

Las externalidades que valorizan el barrio responden a su ubicación en una zona privilegiada de la ciudad. Sin embargo, este aspecto opera también en un sentido negativo: genera amplios contrastes que repercuten en mayores distancias entre los vecinos de la "ciudad formal" y los de la "ciudad informal". Esto refuta la idea de que el acercamiento espacial de agentes muy alejados en el espacio social -argentinos de clase media y migrantes pobres- pueda tener, por sí mismo, un efecto de acercamiento social: "de hecho, nada es más intolerable que la proximidad física (experimentada como promiscuidad) de individuos socialmente muy distantes", sostiene Bourdieu (1999, p.119). La impronta migratoria del barrio estudiado y su lógica doble de inclusión / exclusión son en gran parte responsables por esta distancia.

Pero el barrio no solo es elegido por estas razones instrumentales, sino también por motivos simbólicos. La mayoría de los entrevistados -especialmente los migrantestienen ahí a sus familiares, amigos y paisanos. Este factor tiene importantes implicancias en términos de redes y capital social, que en parte explican la elevada concentración de población de nacionalidad y ascendencia peruana en el barrio.

Los testimonios de los migrantes sudamericanos dan cuenta de los cambios que se han producido en Argentina en el periodo analizado, y que han repercutido

19 Oszlak (1991) define al derecho al espacio urbano como la capacidad de fijar el lugar de residencia o de localización de la actividad económica dentro del espacio, una capacidad que puede ampliarse a la disposición unilateral de los bienes que lo ocupan o a la participación en los procesos de decisión sobre obras de infraestructura y servicios colectivos en espacios públicos o privados adyacentes. Este derecho implica distintas externalidades, estrechamente relacionadas con la localización de la vivienda o la infraestructura económica, como la educación, la recreación, la fuente de trabajo, la atención de la salud, el transporte o los servicios públicos.

20 Esto contrasta con los estudios que señalan una tendencia a la periferización de la pobreza en las metrópolis latinoamericanas (Aguilar \& López, 2016). 
positivamente en sus condiciones de vida. Entre las transformaciones más salientes se cuenta el mayor acceso a la documentación, en el marco de la Ley de Migraciones Nro. 25871 (2004), y particularmente del Programa de Normalización Documentaria Migratoria "Patria Grande" (2005). ${ }^{21}$ Sin embargo, estos cambios no resultan suficientes para revertir las desigualdades que afectan a los migrantes. En efecto, sus testimonios desnudan hasta qué punto sus experiencias en Argentina aún se encuentran fuertemente marcadas por la discriminación, la cual se expresa en distintos ámbitos de la vida social, pero se relaciona especialmente con (e incide en) el plano sociohabitacional.

Debido a la frecuencia y la variedad de experiencias discriminatorias que enfrentan en los distintos ámbitos sociales, los migrantes eligen vivir en barrios como el Playón de Chacarita, donde no se sienten discriminados y diferentes, sino identificados y entre iguales. Las redes de migración no solo permiten el acceso y la movilidad dentro de los barrios, sino que también cumplen una función afectiva: alivianan el sentimiento de desarraigo que azota a las experiencias migratorias. Esta función del barrio popular como comunidad para los migrantes fue señalada por otras investigaciones (Matossian, 2010). Si bien los migrantes son conscientes de los aspectos simbólicos negativos -la exclusión - que comporta el hecho de vivir en una villa, en muchos discursos el barrio aparece también como una forma menos conflictiva de inclusión en la ciudad. Esto nos trae nuevamente a la lógica doble de inclusión / exclusión de la impronta migratoria del barrio. La identificación que los migrantes sienten "dentro" del barrio tiene su contrapunto en el rechazo que enfrentan "afuera". Los cambios en los rasgos estructurales y, fundamentalmente, en la composición migratoria de la población de este espacio traen aparejada una mayor distancia social con el entorno social inmediato (el barrio) y más amplio (la ciudad).

\section{Entre la exclusión y la inclusión}

A través del análisis de los hallazgos de un estudio de caso en la ciudad de Buenos Aires, en diálogo con la bibliografía especializada, este artículo se propuso brindar elementos para la reflexión sobre las articulaciones entre procesos migratorios y dinámicas de la informalidad urbana que se producen en el periodo 2001-2014, y sus efectos en términos de producción de lógicas de exclusión e inclusión.

El trabajo propone una reflexión general sobre la impronta migratoria de los hábitats precarios de la САВA. Esta impronta es el resultado de las nuevas articulaciones que se producen entre los procesos migratorios y las dinámicas de la informalidad urbana en el periodo analizado, pero también de las imbricaciones pasadas entre estos fenómenos, en las que también interviene la acción del Estado. El artículo pretende mostrar cómo se actualiza el histórico entrelazamiento entre los procesos de exclusión basados en la clase social y en el origen étnico-nacional, y cómo se configura un

21 Según datos del programa, entre 2006 y 2009 se inscribieron 423.697 personas, y se otorgaron 98.539 radicaciones permanentes y 126.385 radicaciones temporarias. Véase Ministerio del Interior, Dirección Nacional de Migraciones (2010). 
nuevo "problema": las villas pobladas por migrantes, enclaves de otredad por excelencia en la ciudad del siglo xxI y, por ende, depositarias de miedos y acusaciones.

Los conflictos que se suceden en este periodo son tomados como problemas sociológicos por las ciencias sociales, que comienzan a producir investigaciones centradas en esta particular intersección entre procesos migratorios y dinámicas de la informalidad urbana. La emergencia de este problema sociológico confirma el rango de "problema social" que dicha intersección ha venido afianzando desde varias décadas atrás. El artículo entrega un conjunto de datos estadísticos que permiten comenzar a dimensionar la realidad migratoria en los distintos hábitats precarios de la ciudad. Por un lado, se señala que los migrantes se encuentran más fuertemente representados en estos espacios que en el "resto de la ciudad": un dato que legitima la realización de investigaciones como esta. Por otro lado, se introducen datos que permiten refutar los discursos que vinculan procesos migratorios e informalidad urbana de forma espuria, argumentando que: (i) los migrantes no constituyen la mayor parte de la población de los hábitats precarios; y (ii) la mayoría de los migrantes no se inserta residencialmente en hábitats precarios.

Como apartado central del artículo se analizan las experiencias de los migrantes que residen en el Playón de Chacarita. Se describe el contexto del arribo de los primeros migrantes a este barrio: un escenario signado por relativas mejoras económicas a nivel nacional, políticas habitacionales de emergencia (subsidios) a nivel local, el surgimiento de un mercado inmobiliario informal en este y otros espacios precarios, y oportunidades - no exentas de conflictos- para las ocupaciones de terrenos. El estudio permite una aproximación a la configuración de un escenario doblemente excluyente para el acceso a la vivienda de los migrantes, uno que los excluye por motivos tanto socioeconómicos como étnico-nacionales.

El trabajo reflexiona sobre las trayectorias residenciales de los habitantes de este barrio, subrayando que ellas se encuentran marcadas por la precariedad, la informalidad, la inseguridad y la inestabilidad. También establece que estas trayectorias resultan de una particular conjunción de elementos: una articulación de modalidades informales de inserción en los mercados laboral e inmobiliario, un conjunto de aprendizajes adquiridos y reproducidos en el marco de dichas trayectorias, una extendida expectativa de convertirse en propietarios de una vivienda y del suelo en el que se ubica, y una fuerte incidencia de los recursos sociales en las estrategias de supervivencia de estos sectores.

También se examina el contexto político de la CABA a partir de 2007, cuando da inicio la primera administración macrista. No solo se señala la ausencia de herramientas adecuadas para tratar la problemática habitacional que afecta a la CABA, sino también la creciente fragmentación de las políticas destinadas a solucionar dicha cuestión.

Finalmente, se analiza la incidencia de los recursos sociales en estos procesos: se muestra su papel central en la composición poblacional del barrio y en las formas de sociabilidad que allí se desarrollan. El trabajo reflexiona sobre los vínculos de los migrantes con el funcionamiento del mercado inmobiliario informal en las villas, la relevancia de la estrategia de cohabitación, las formas concretas en que acceden a estos barrios, y el dinamismo que caracteriza a los recursos que aplican. Explica 
el lugar de tales recursos en la configuración de la impronta migratoria de estos barrios, y la lógica doble de inclusión / exclusión que ella implica.

Para concluir, se indaga en los sentidos que los propios habitantes le atribuyen a la vida en el barrio. El artículo reflexiona sobre el proceso de resignificación parcial de los barrios precarios al que se asiste, así como sobre la disputa simbólica que la consolidación de este espacio habilita. También describe las razones instrumentales y simbólicas que fundamentan la elección de este lugar de residencia por parte de los migrantes. Desde la perspectiva instrumental, el barrio es elegido por sus externalidades, garantizadas por su ubicación en una zona jerarquizada de la ciudad: una ubicación que, no obstante, les acarrea una fuerte distancia social respecto de su entorno. Desde el punto de vista simbólico, el barrio no solo aparece como una posibilidad de estabilidad y seguridad, sino que también es visto como un espacio de inclusión, que permite enfrentar las diversas formas de exclusión que afectan a esta población en otros ámbitos sociales.

En definitiva, este artículo se propuso contribuir a los debates sobre algunas de las dinámicas de la exclusión y la inclusión que actualmente conviven en la ciudad de Buenos Aires. La histórica construcción simbólica de los procesos migratorios y de las villas como problemas sociales desemboca en la alterización de estos grupos. Ello se refleja en diversas formas de desigualdad con correlatos territoriales (configuraciones socioespaciales como el Playón de Chacarita, que se multiplican en el mapa) y simbólicos (discursos que anudan estos fenómenos de forma espuria, contribuyendo así a la subordinación de los grupos afectados). Estas desigualdades atestiguan los rasgos contemporáneos de las imbricaciones entre los distintos procesos de alterización (por clase social y por origen étnico-nacional) y sus consecuencias en las condiciones de vida de amplios sectores de la población. Sin dudas, se trata de un importante desafío para la formulación de políticas públicas comprometidas con el respeto de los derechos humanos de todas las personas que habitan en Argentina, especialmente de cara a un nuevo ciclo político en el país, que promete profundizar algunas de las tendencias aquí reseñadas.

\section{Referencias bibliográficas}

Abramo, P. (Org.) (2003). A cidade da informalidade: os desafios das cidades latino-americanas. Rio de Janeiro: Sette Letras-Faperj-Lincoln Institute.

Aguilar, A. \& López, F. (2016). Espacios de pobreza en la periferia urbana y suburbios interiores de la Ciudad de México. Las desventajas acumuladas. EURE, 42(125), 5-29. https:// doi.org/10.4067/S0250-71612016000100001

Arias, G., Moreno, R. \& Núñez, D. (2010). Inmigración latinoamericana en Chile: analizando perfiles y patrones de localización de la comunidad peruana en el Área Metropolitana de Santiago (Aмs). Tiempo y Espacio, 21(25), 59-77. http://revistas.ubiobio.cl/index. $\mathrm{php/TYE/article/view/1769}$ 
Baer, L. (2008). Crecimiento económico, mercado inmobiliario y ausencia de política de suelo. Un análisis de la expansión del espacio residencial de la Ciudad de Buenos Aires en los 2000. Proyección, 2(5), s/p. http://bdigital.uncu.edu.ar/3256

Baltrusis, N. (2004). Mercado imobiliário informal em favelas na região metropolitana de São Paulo. O caso de Guarulhos. Cadernos Metrópole, (11), 107-136. http://www.fau.usp. $\mathrm{br} /$ depprojeto/labhab/biblioteca/textos/baltrusis_mercadoinformal.pdf

Beccaria, L., Maurizio, R. \& Esquivel, V. (2005). Empleo, salarios y equidad durante la recuperación reciente en la Argentina. Desarrollo Económico, Revista de Ciencias Sociales, 45(178), 235-262. doi: 10.2307/3655858

Bellardi, M. \& De Paula, A. (1986). Villas Miseria: origen, erradicación y respuestas populares. Buenos Aires: Centro Editor de América Latina.

Benencia, R. (2005). Redes sociales de migrantes limítrofes: lazos fuertes y lazos débiles en la conformación de mercados de trabajo hortícola (Argentina). Ponencia presentada al $7^{\circ}$ Congreso Nacional de Estudios del Trabajo, realizado entre el 10 y el 12 de agosto en la Universidad de Buenos Aires (Argentina). http://www.aset.org.ar/congresos/7/15011.pdf

Benencia, R. (2012). Migraciones y situaciones en el ámbito del trabajo. Voces en el Fénix, 3(21), 12-17. http://www.vocesenelfenix.com/category/ediciones/n\%C2\%BA-21

Bourdieu, P. (Dir.) (1999). Efectos de lugar. En P. Bourdieu, La miseria del mundo (pp. 119-124). Madrid: Akal.

Caggiano, S., Casullo, M. E., Cravino, M. C., Cremonte, J. P., Fernández Wagner, R., Grimson, A., Kaufman, A., López (...) \& Vommaro, G. (2012). Racismo, violencia y politica. Pensar el Indoamericano, dos años después. Colección Cuestiones Metropolitanas, 7. Los Polvorines: Universidad Nacional de General Sarmiento.

Canelo, B. (2013). Fronteras internas. Migración y disputas espaciales en la Ciudad de Buenos Aires. Buenos Aires: Antropofagia.

Centro de Estudios Legales y Sociales (CELs). (2009). Derechos humanos en la Argentina: Informe 2009. Buenos Aires: Siglo XxI.

Cerrutti, M. \& Grimson, A. (2004). Buenos Aires, neoliberalismo y después. Cambios socioeconómicos y respuestas populares. Serie Cuadernos del IDES, 5. Buenos Aires: Instituto de Desarrollo Económico y Social. http://ides.org.ar/wp-content/ uploads/2012/03/Cuaderno5_Cerrutti_Grimson.pdf

Clichevsky, N. (2003). Territorios en pugna: las villas de Buenos Aires. Revista Ciudad y Territorio. Estudios Territoriales, 35(136-137), 347-374. https://ecaths1.s3.amazonaws. com/.../13778245.Villas\%20Clichevsky.pdf

Cravino, M. (2006). Las villas de la Ciudad. Mercado e informalidad urbana. Los Polvorines: Universidad Nacional de General Sarmiento.

Cravino, M. (2008). Relaciones entre el mercado inmobiliario informal y las redes sociales en asentamientos informales en el Área Metropolitana de Buenos Aires. Territorios, (1819), 129-145. http://www.redalyc.org/pdf/357/35711626006.pdf.

Cravino, M. (2011). El ciclo de las villas y el mercado inmobiliario informal. Voces en el Fénix, 1(5), 31-33. http://www.vocesenelfenix.com/sites/default/files/pdf/5cravino_5.pdf

Cravino, M. (2012). La rebelión de los inquilinos. Procesos migratorios y ciudad informal. En S. Caggiano et al., Racismo, violencia y politica. Pensar el Indoamericano, dos años después (pp. 127-154). Colección Cuestiones Metropolitanas, 7. Los Polvorines: Universidad Nacional de General Sarmiento. 
Di Filippo, F. (2010). Buenos Aires sin techo. Informe sobre la emergencia habitacional en la Ciudad de Buenos Aires. Buenos Aires: Comisión de Vivienda, Legislatura de la Ciudad de Buenos Aires.

Di Virgilio, M. (2007). Trayectorias residenciales y estrategias habitacionales de familias de sectores populares y medios en Buenos Aires. Tesis de posgrado, Doctorado en Ciencias Sociales, Facultad de Ciencias Sociales, Universidad de Buenos Aires.

Di Virgilio, M. (2012). Participación social y organizaciones sociales en la implementación de políticas orientadas a la producción social del hábitat en el Área Metropolitana de Buenos Aires. Revista SAAP (Sociedad Argentina de Análisis Político) [en línea], 6(1). http:// www.scielo.org.ar/scielo.php?script=sci_arttext\&pid=S1853-19702012000100001

Di Virgilio, M. \& Gil y de Anso, M. L. (2012). Estrategias habitacionales de familias de sectores populares y medios residentes en el área metropolitana de Buenos Aires (Argentina). Revista de Estudios Sociales, (44), 158-170. https://dialnet.unirioja.es/ servlet/articulo? codigo $=4111300$

Dimarco, S. (2007). ¿Podremos mirar más allá de la basura? Raneros, cirujas y cartoneros: historias detrás de la basura. Papeles del CEIC, 2(33), 2-29. https://dialnet.unirioja.es/ servlet/articulo?codigo $=2379904$

Domenech, E. (Comp.) (2009). Migración y política: el Estado interrogado. Procesos actuales en Argentina y Suramérica. Córdoba: Universidad Nacional de Córdoba.

Encuesta Anual de Hogares (ЕАн) 2012. Véase Ministerio de Hacienda, Dirección General de Estadística y Censos, Argentina (2013).

Ferraudi Curto, M. (2011). Las políticas habitacionales post-2001 desde una villa del Gran Buenos Aires: el Estado en construcción. Trabajo y Sociedad, 15(17), 123-138. http:// www.scielo.org.ar/scielo.php?script=sci_arttext\&pid=S1514-68712011000200010

Gago, V. \& García Pérez, E. (2014). Ciudad próspera, ciudad monstruosa: nuevas racionalidades urbanas a partir del caso Indoamericano. Quid 16, 4, 66-83. http://publicaciones. sociales.uba.ar/index.php/quid16/article/view/1152

Gallinati, C. \& Gavazzo, N. (2011). Nacionales y extranjeros frente al déficit habitacional: modalidades de acceso a la vivienda y lucha por la propiedad de la tierra en el Área Metropolitana de Buenos Aires. Temas de Antropología y Migración, Dossier (especial): El Parque Indoamericano en diciembre de 2010: otras tramas, otras miradas, (1), 37-55. http://www.migrantropologia.com.ar/images/stories/PDF/Revista1/Articulo5.pdf

Garcés, A. (2010). De enclave a centralidad. Espacio urbano, comercio y migración peruana en Santiago de Chile. Gazeta de Antropología, 27(2), s/p. http://www.ugr.es/-pwlac/ G27_38Alejandro_Garces.html

Grimson, A. (2009). Articulaciones cambiantes de clase y etnicidad: una villa miseria de Buenos Aires. En A. Grimson, C. Ferraudi Curto \& R. Segura, R. (Comps.), La vida política en los barrios populares de Buenos Aires (pp. 221-147). Buenos Aires: Prometeo.

Grimson, A. (2011). Relatos de la diferencia y la igualdad. Buenos Aires: Eudeba.

Grimson, A. \& Caggiano, S. (2012). Xenofobias descontroladas. En S. Caggiano et al., Racismo, violencia y política. Pensar el Indoamericano, dos años después (pp. 61-78). Los Polvorines: Universidad Nacional de General Sarmiento.

Gutiérrez, A. (2008). Redes e intercambio de capitales en condiciones de pobreza: dimensión relacional y dimensión vincular. REDES Revista Hispana para el análisis de las redes sociales, 14(4), s/p. http://www.redalyc.org/articulo.oa?id=93101404 
Halpern, G. (2009). Etnicidad, inmigración y politica. Buenos Aires: Prometeo.

Hintze, S. (2004). Capital social y estrategias de supervivencia. Reflexiones sobre el capital social de los pobres. En C. Danani (Comp.), Políticas sociales y economía social: debates fundamentales (pp. 143-166). Buenos Aires: Universidad Nacional de General Sarmiento.

Jiménez Huerta, E. (2000). El principio de la irregularidad. Mercado del suelo para vivienda en Aguascalientes, 1975-1998. Guadalajara, México: Universidad de Guadalajara.

Lekerman, V. (2005). Procesos informales de ocupación de tierras en la Ciudad de Buenos Aires. ¿Villas o asentamientos? El caso del asentamiento Costanera Sur. Los excluidos del sueño. Cuadernos de Antropología Social, (22), 115-135. http://www.redalyc.org/ articulo.oa?id=180913913007

Luque, J. (2013). Transnacionalismo y enclave territorial étnico en la configuración de la ciudadanía de los migrantes peruanos en Santiago de Chile. Revista Enfoques: Ciencia Politica y Administración Pública, 3(4), 81-102.

Madero, I. \& Mora, C. (2011). Capital social e inclusión laboral. Una aproximación a las trayectorias de ascendencia laboral de migrantes peruanos en Chile. Polis Revista Latinoamericana, 10(29), 147-163. https://doi.org/10.4067/S0718-65682011000200007

Maguid, A. \& Arruñada, V. (2005). El impacto de la crisis en la inmigración limítrofe y del Perú hacia el Área Metropolitana de Buenos Aires. Revista Estudios del Trabajo, (30), 95-121. http://www.aset.org.ar/docs/Maguid\%20Arrunada\%2030.pdf

Matossian, B. (2010). Expansión urbana y migración. El caso de los migrantes chilenos en San Carlos de Bariloche como actores destacados en la conformación de barrios populares. Scripta Nova, 14(331-76). http://www.ub.edu/geocrit/sn/sn-331/sn-331-76.htm

Mera, G. (2012). Migración y espacio urbano. Distribución de los migrantes paraguayos en la Ciudad de Buenos Aires: procesos de diferenciación y segregación espacial. Tesis de posgrado, Doctorado en Ciencias Sociales, Facultad de Ciencias Sociales, Universidad de Buenos Aires.

Mera, G. \& Marcos, M. (2015). Cartografías migratorias urbanas. Distribución espacial de la población extranjera en la Ciudad de Buenos Aires (2010). Geograficando, 11(1), s/p. http://www.geograficando.fahce.unlp.edu.ar/article/view/Geov11n01a04

Mera, G. \& Vaccotti, L. (2013). Migración y déficit habitacional en la Ciudad de Buenos Aires: resignificando el "problema”. Argumentos. Revista de Crítica Social, (15), 176-202. http://publicaciones.sociales.uba.ar/index.php/argumentos/article/viewFile/906/792

Merklen, D. (1999). La cuestión social en el sur desde la perspectiva de la integración. Políticas sociales y acción colectiva en los barrios marginales del Rio de la Plata. Serie Documentos de Políticas Sociales, 20. Buenos Aires: Secretaría de Promoción Social.

Ministerio de Hacienda, Dirección General de Estadística y Censos, Argentina (2013, noviembre). Encuesta Anual de Hogares 2012. Estructura de la población. Informe de resultados 601. Buenos Aires: Autor. https://www.estadisticaciudad.gob.ar/eyc/wpcontent/uploads/2015/04/ir_2013_601.pdf

Ministerio del Interior, Dirección Nacional de Migraciones, Argentina (2005). Disposición 53253/2005: Programa Nacional de Normalización Documentaria Migratoria para los extranjeros de los Estados Parte del mercosur y de sus Estados Asociados (Programa Patria Grande). Publicado en el Boletín Oficial del 15 de diciembre de 2005. https://www.oas. org/dil/Migrants/Argentina/Disposici\%C3\%B3n\%2014.949-2006.pdf 
Ministerio del Interior, Dirección Nacional de Migraciones, Argentina (2010). Patria Grande. Programa Nacional de Normalización Documentaria Migratoria. Informe Estadístico, agosto 2010. http://www.migraciones.gov.ar/pdf_varios/estadisticas/Patria_Grande.pdf

Novick, S. (Comp.). Con la colaboración de C. Stefoni y A. Hinojosa Gordonava (2008). Las migraciones en América Latina. Politicas, culturas y estrategias. Buenos Aires: Consejo Latinoamericano de Ciencias Sociales (Clacso) / Catálogos / Agencia Sueca de Desarrollo Internacional (ASDI).

Oszlak, O. (1991). Merecer la ciudad: los pobres y el derecho al espacio urbano. Buenos Aires: Centro de Estudios de Estado y Sociedad (CEDEs) / Hvmanitas.

Pedone, C. (2003). "Tú siempre jalas a los tuyos". Cadenas y redes migratorias de las familias ecuatorianas hacia España. En G. Herrera, M. C. Carrillo \& A. Torres (Eds.), La migración ecuatoriana. Transnacionalismo, redes e identidades (pp. 105-143). Quito: Facultad Latinoamericana de Ciencias Sociales (FLACso-Ecuador). http://www. flacsoandes.edu.ec/libros/17875-opac

Rodríguez, M. (2005). Como en la estrategia del caracol... Ocupaciones de edificios y politicas locales del hábitat en la ciudad de Buenos Aires. Buenos Aires: El Cielo por Asalto.

Rodríguez, M., Arqueros, S., Rodríguez, M. F., Gómez, M. \& Zapata, M. C. (2011). La política urbana "PRO": continuidades y cambios en contextos de renovación en la Ciudad de Buenos Aires. Cuaderno Urbano, 11(11), 101-121. http://revistas.unne.edu.ar/index. $\mathrm{php} / \mathrm{cua} /$ article/view/568

Rosas, C. (2010). Implicaciones mutuas entre el género y la migración. Mujeres y varones peruanos arribados a Buenos Aires entre 1990 y 2003. Buenos Aires: Eudeba.

Rosas, C., Corbetta, S. \& Toledo, J. M. (2013). Migraciones y procesos de inserción social en asentamientos del conurbano bonaerense argentino. Ponencia presentada al I Congreso internacional Nuevos horizontes de Iberoamérica, realizado entre el 6 y el 8 de noviembre de 2013 en la Universidad Nacional de Cuyo (Argentina).

Sassone, S. (2007). Migración, territorio e identidad cultural: construcción de "lugares bolivianos" en la Ciudad de Buenos Aires. Población de Buenos Aires, 4(6), 9-28. http:// www.redalyc.org/articulo.oa?id=74040601

Sassone, S. \& Mera, C. (2007). Barrios de migrantes en Buenos Aires: Identidad, cultura y cohesión socioterritorial. Ponencia presentada al $V$ Congreso Europeo CEISAL de latinoamericanistas, realizado entre el 11 y el 14 de abril en CERCAL [Centro de Estudios y de Promoción de las Relaciones entre los países de la Unión Europea y América Latina]. Université Libre de Bruxelles (Bélgica).

Segura, D. \& Abde, K. (2014). Barrios y población inmigrantes: el caso de la comuna de Santiago. Revista INVI, 29(81), 19-77. doi: 10.4067/S0718-83582014000200002

Szajnberg, D. (2009). Los Nuevos Asentamientos informales en la Ciudad de Buenos Aires. El caso de las tierras desactivadas en los barrios de Chacarita, Paternal, Caballito y Barracas. Buenos Aires: Nobuko.

Vaccotti, L. (2014). En los márgenes de la politica. Migrantes y movilizaciones por el derecho a la vivienda en las villas de la ciudad de Buenos Aires: el caso del Playón de Chacarita (2001-2014). Tesis de Doctorado en Ciencias Sociales, Facultad de Ciencias Sociales, Universidad de Buenos Aires (Argentina).

Vaccotti, L. (2017). La judicialización de la lucha por la vivienda en Buenos Aires. ERLACS, (103), 51-70. doi: http://doi.org/10.18352/erlacs.10142 